\title{
Effects of hydrogenation on electrical properties of InP grown on GaAs by the photochemical vapor deposition system
}

\author{
T. Y. Lin, Y. F. Chen \\ Physics Department, National Taiwan University, Taipei (Taiwan, ROC) \\ W. K. Chen \\ Electrophysics Department, National Chiao Tung University, Hsinchu (Taiwan, ROC) \\ and Y. S. Lue \\ Center of precision Measurement, Industrial Technology Research Institute, Hsinchu (Taiwan, ROC)
}

(Received April 22, 1992; accepted June 8, 1992)

\begin{abstract}
Effects of hydrogenation on electrical properties of InP epilayers grown on GaAs substrates by 'photochemical vapor deposition' have been investigated. The electrical properties were characterized by current-voltage and capacitance-voltage measurements at room temperature. Deep level transient spectroscopy technique was used as an auxiliary method to detect the deep level traps. Hydrogen plasma exposure at $250{ }^{\circ} \mathrm{C}$ for $3 \mathrm{~h}$ increased the reverse breakdown voltage by a factor of about 1.7 and the Schottky barrier height by $0.05 \mathrm{eV}$. In addition, the shallow and deep levels were substantially passivated. These results indicate that hydrogenation by the photochemical vapor deposition system for InP on $\mathrm{GaAs}$ is an excellent method to improve its device application.
\end{abstract}

Fabrication of high performance, multifunctional optoelectric circuits and devices is the aim for many novel growth techniques. Recent attention has been given to the epitaxial combination of materials with different lattice constants. Currently, the combination of InP and GaAs based materials with silicon or lattice mismatched III-V substrates ( $\mathrm{GaAs}$ and $\mathrm{InP}$ ) has evoked much interest because of the well-established technology of GaAs. It is advantageous to integrate high speed GaAs electronic devices with InP based optical devices on a single substrate. In order to achieve this integration, the $\mathrm{InP} / \mathrm{GaAs}$ system is preferable to the GaAs/InP system, because large area and low cost GaAs substrates are available. Recently, highquality epitaxial films of InP/GaAs were grown by molecular-beam epitaxy [1] (MBE), metal organic chemical vapor deposition [2-4] (MOCVD) and their structural variations on thermal treatment were investigated succeedingly [5]. A common feature of such heteroepitaxial devices is the formation of relaxed layers due to the large lattice mismatch $(3.8 \%)$ between the epilayers and substrates which result in the relatively high reverse leakage currents in the simple diode or transistor structures fabricated on this material. Swaminathan and Macrander [6] have shown from a double crystal diffractometer study that a InP layer grown by MOCVD on a GaAs substrate has poor crystallinity. It thus appears that further insight into the related physical properties of this material will provide great improvements for the interest of designs and applications.

Among a variety of schemes which are known to reduce the high density of dislocations and the deleterious effect of the consequent defects, the post growth hydrogenation method is being used frequently. The passivation effect of incorporation of atomic hydrogen into semiconductors has become more valid through the study of hydrogenated amorphous silicon (a-Si:H) [7]. Its ability to produce remarkable electrical and optical changes has attracted wide interest in recent years. There are extensive reviews on this topic written by Pearton et al. [8] and by Haller [9]. Recently, a number of groups have reported the effects of post growth hydrogenation of GaAs on $\mathrm{Si}[10,11]$ and GaAs on InP [11-13]. In this paper, we report on the substantial changes in the current-voltage and capacitance-voltage characteristics of InP grown on GaAs substrates as a result of post plasma hydrogenation for the first time. 
Plasma exposure was performed in a photochemical vapor deposition system. This is an alternative method we have recently developed [14] for the injection of atomic hydrogen into crystalline semiconductors without ion and electron bombardment. And this technique was demonstrated to passivate also the damaged surface during hydrogenation using RF glow-discharge method [15]. For the photochemical vapor deposition system, the ultraviolet light from a low-pressure Hg lamp was employed as the light source. The mercury photosensitization method was used to enhance the dissociation of reactant gas $\mathrm{H}_{2}$. The $\mathrm{H}_{2}$ gas could be premixed with a small amount of mercury vapor in a thermally controlled mercury vaporizer, before being introduced into the reactor. A detailed description of the apparatus has been described elsewhere [14]. Here, we present the effects of the incorporation of atomic hydrogen into InP grown on GaAs substrates by the photochemical vapor deposition system.

Passivation conditions are as follows: hydrogen flow rate, 40-50 sccm; chamber pressure, 1-2 mbar; sample temperature, $250{ }^{\circ} \mathrm{C}$; the temperature of $\mathrm{Hg}$ container, $50{ }^{\circ} \mathrm{C}$; exposure time, $3 \mathrm{~h}$.

The InP epitaxial layers were grown by MOCVD in the Si doped [100] polar GaAs substrates. Typical growth conditions are a growth temperature of $610{ }^{\circ} \mathrm{C}$ under atmospheric pressure and a ratio of $\mathrm{PH}_{3}$ to triethylindium (TEIn) of 110. The thickness of the InP epilayer is about $4.3 \mu \mathrm{m}$. Characterization measurements were done with Schottky barrier diodes. For all samples, an Au:Ge/ Ni ohmic contact was deposited by thermal evaporation under a high vacuum, and annealed at $410{ }^{\circ} \mathrm{C}$ with $1.5 \mathrm{lpm}$ forming gas for 7 mins. A layer of photo resist was used to protect the ohmic contact during acidic cleaning. The front surfaces were first etched in sulfuric acid solution and then in dilute HF (49\%) solution. Finally, the $\mathrm{Au}$ Schottky contact was formed in a high vacuum chamber. The Schottky junctions were evaluated using measurements of forward or reverse $I-V$ characteristics and $C-V$ profile in a light-tight box at room temperature.

In a previous report [16], using deep-level-transient spectroscopy (DLTS) technique we have shown that hydrogenation can effectively reduce both the interface and bulk defects for InP grown on GaAs substrates. Here, to complete our discussion, we present the corresponding DLTS data. Figure 1(a) shows the typical DLTS spectra of the InP epitaxial layer before and after hydrogenation. It is evident that the two observed electron traps, $E_{1}$ and $E_{2}$, with the activation energy,
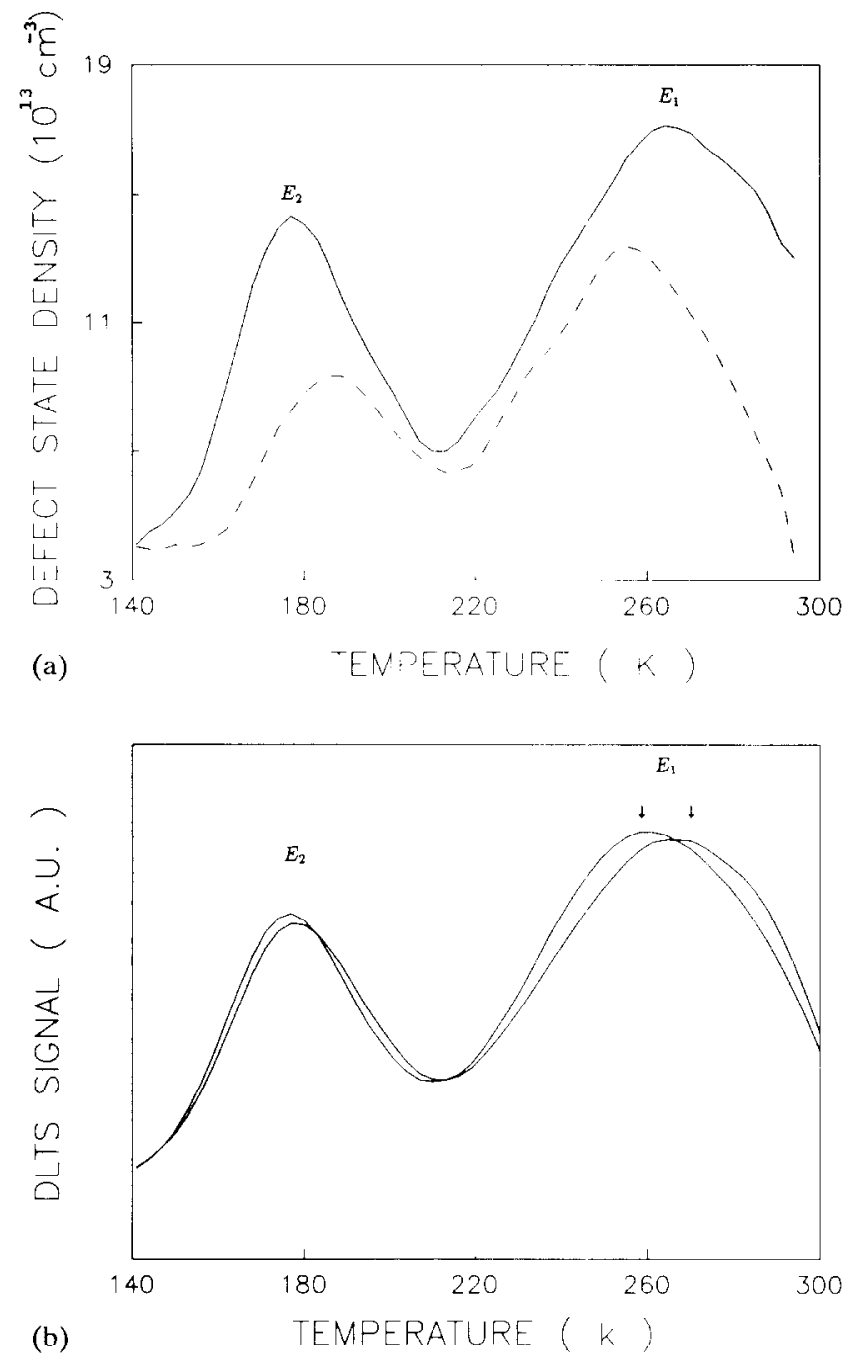

Fig. 1. (a), DLTS spectra of $n$-InP epitaxial layers on GaAs substrates before (solid line) and after (dashed line) hydrogenation. (b), DLTS spectra of $n$-InP epitaxial layers on GaAs substrates. The characteristic shift in the temperature position clearly shows the interface origin of trap $E_{1}$ at $E_{1}=0.46 \mathrm{eV}$. Pulse width: $0.04 \mathrm{~s}$; pulse height: $0.3 \mathrm{~V}, 0.4 \mathrm{~V}$; rate window: 73 ms.

$E_{\mathrm{t}}-E_{\mathrm{c}}=0.46 \mathrm{eV}$ and $0.28 \mathrm{eV}$ respectively, were effectively passivated by the hydrogenation process. It is reported [17] that the distinction between peaks due to bulk traps and peaks due to interface traps can be verified by varying the filling pulse $V_{\mathrm{f}}$ and/or reverse bias $V_{\mathrm{R}}$. The temperature position of peaks due to bulk traps remains unchanged with either $V_{\mathrm{f}}$ or $V_{\mathrm{R}}$, while peaks due to interface traps show significant shift in positions. Figure 1(b) shows that the peak position of trap $E_{1}$ shifted with the change of the filling pulse height which identifies it as a surface trap. In contrast, the peak position of trap $E_{2}$ does not change with the filling pulse, we thus can attribute this peak to a native defect in the heteroepitaxially grown layer. There- 
fore, we have demonstrated that the incorporation of atomic hydrogen can successfully passivate surface and native defects in InP on the GaAs heterojunction.

Figure 2(a) shows reverse current-voltage (I-V) curves from untreated and hydrogenated diodes. There is a substantial increase in the reverse breakdown voltage in the treated Schottky junction. We adopted the convention that this is the voltage at which the reverse leakage current is $1 \mathrm{~mA}$. Figure 2(b) shows the forward $I-V$ characteristics from the various diodes. Data is analyzed using the general expression based on a thermionic emission mechanism [18]. Neglecting the tunneling effects and barrier lowering due to the Schottky effect, and for $\mathrm{k} T / \mathrm{q} \ll V$, the dependence of the current density on the applied voltage $V$ is given by

$J=\mathrm{A}^{* *} T^{2} \exp \left(-\phi_{\mathrm{B}} / \mathrm{k} T\right)[\exp (\mathrm{q} V / n \mathrm{k} T)]$

where $J$ is the current density, $\mathrm{A}^{* *}$ is the effective Richardson constant, $\mathrm{A}^{* *}=\mathrm{A}^{*}\left(m^{*} / m_{0}\right), T$ is the sample temperature, $\phi_{\mathrm{B}}$ is the effective Schottky barrier height, $\mathrm{k}$ is the Boltzmann constant, $\mathrm{q}$ is the electronic charge, and $n$ is the ideality factor. Assuming $\mathrm{A}^{*}=120 \mathrm{Acm}^{-2} \mathrm{k}^{-2}$ and $\mathrm{m}^{*} / m_{0}=0.077$ [19] for InP, the theoretical value of $A^{* *}$ is approximately $9.2 \mathrm{Acm}^{-2} \mathrm{k}^{-2}$. Table 1 shows the

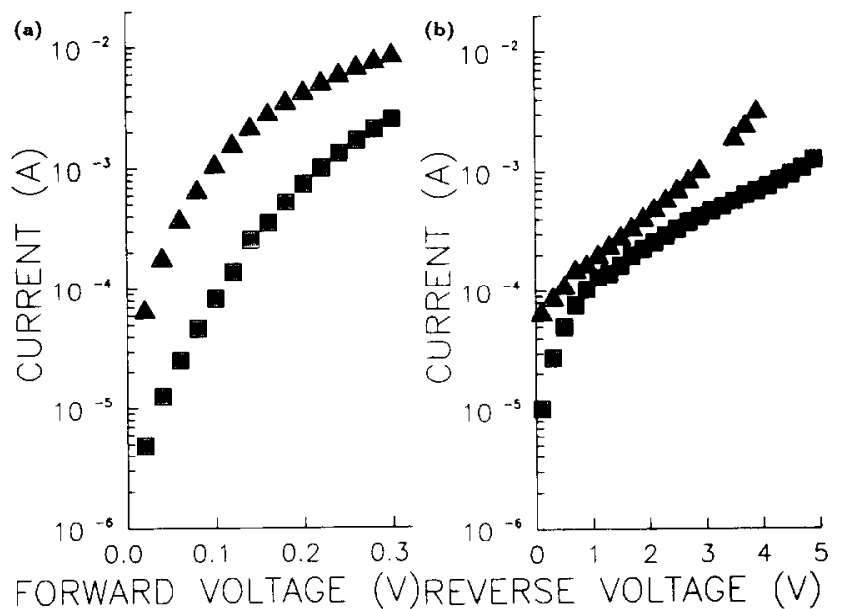

Fig. 2. Current voltage characteristics of $n$-InP epitaxial layer on GaAs substrates before $(\triangle)$ and after $(\square)$ hydrogenation. Part (a) refers to the reverse current results and part (b) to forward current results.

TABLE 1. $I_{0}(\mathrm{~A})$ : saturation current, $n$ : ideality factor; $\phi_{\mathrm{B}}(\mathrm{eV})$ : barrier height; $V_{\mathrm{B}}(\mathrm{V})$ : break-down voltage for the InP on GaAs Schottky diode before and after hydrogenation

\begin{tabular}{lllll}
\hline Sample & $I_{\mathrm{U}}(\mathrm{A})$ & $n$ & $\phi_{\mathrm{B}}(\mathrm{eV})$ & $V_{\mathrm{B}}(\mathrm{V})$ \\
As-grown & $4.14 \times 10^{-5}$ & 1.13 & 0.46 & 2.7 \\
Hydrogenated & $4.33 \times 10^{-6}$ & 1.10 & 0.51 & 4.6 \\
\hline
\end{tabular}

intercept saturation currents, ideality factors, barrier heights, and breakdown voltages extracted from the $I-V$ data for the sample without and with hydrogenation. The barrier height of the as-grown Schottky junction is comparable with that of the conventional InP Schottky junction [20]. The values of the hydrogenated sample are obtained using the same $A^{* *}$ as that for untreated sample. We can see clearly that the barrier height is increased and the ideality factor tends to approach unity after hydrogenation.

To explain the improvement of the quality of the Schottky diode after hydrogenation as shown above, we may get support from the DLTS measurement. It has been found that the $I-V$ characteristics are insensitive to the metal contact used in GaAs and InP [21]. This phenomenon is believed to be due to the effect of Fermi level pinning caused by surface defects. From the DLTS measurements as shown in Fig. 1, the interfacial and bulk defects can be reduced by hydrogenation, such that the effect of Fermi level pinning is lifted. Therefore, it is expected that hydrogen treatment will change the barrier height. It has also been found that there is a high density of threading dislocations in the heteroepitaxial samples. A likely explanation for the improvement in the breakdown voltage in a plasma treated diode is therefore the passivation by hydrogenation of dangling or defective bonds associated with these dislocations that contribute to excess leakage current in diode structure.

The room temperature $1 / \mathrm{C}^{2}$ versus reverse voltage plot of the as-grown and hydrogenated InP epitaxial layer obtained from $1 \mathrm{MHz}$ capacitancevoltage profile measurements are presented in Fig. 3. The plot shows a slight discrepancy from a

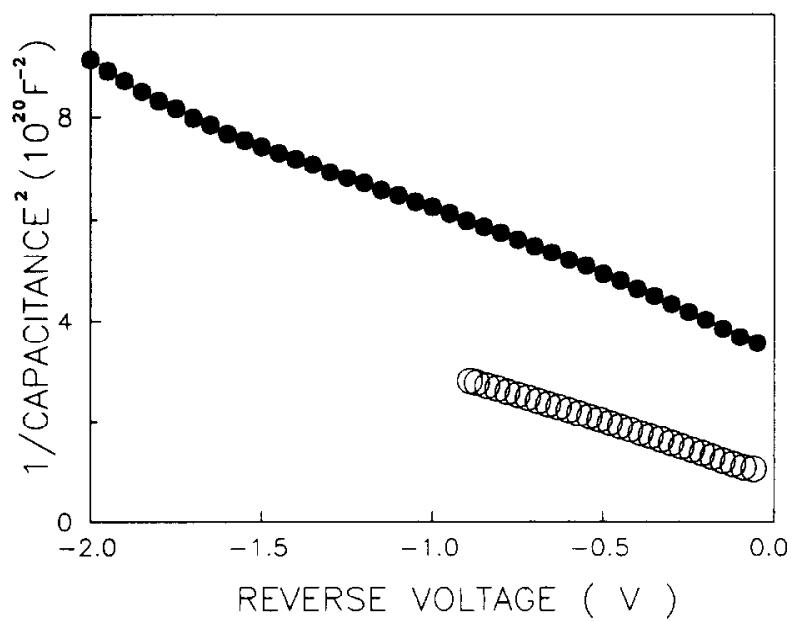

Fig. 3. 1/Capacitance ${ }^{2}$ plots at room temperature of $n$-InP epitaxial layer GaAs substrates before $(O)$ and after $(\bullet)$ hydrogendion. 
linear behavior for the hydrogenated device. The carrier concentration was reduced to $4.8 \times 10^{15}$ $\mathrm{cm}^{-3}$ in the first $5100 \AA$ from the surface, followed by a rise in the carrier profile further into the layer, whereas the corresponding value for the unhydrogenated device is higher at $8.4 \times 10^{15} \mathrm{~cm}^{-3}$. The exact magnitude closer to the surface cannot be determined due to the large space-charge width at zero applied bias, which provides further evidence for a highly passivated surface layer. This suggests that incorporation of atomic hydrogen can produce the donor neutralization. It is generally accepted that the passivation results from hydrogen being attached to the donor in an interstitial antibonding position accepting the extra electron that the donor would normally contribute to the conduction band. Combining with the previous investigation, an immediate consequence of this observation is the inference that hydrogen passivation of shallow impurities is a general physical phenomenon in both elemental and compound semiconductors [8].

\section{Conclusions}

We have used $I-V$ and $C-V$ measurements to investigate the effects of the hydrogenation on the InP grown on GaAs substrates by MOCVD. $I-V$ studies showed an increase of reverse breakdown voltage in heteroepitaxial samples by a factor of about 1.7 and an enhancement of the barrier height related to the reduction of interfacial and bulk defects. The $C-V$ profile showed passivation of shallow donor after hydrogenation. Thus, we find conclusively that hydrogenation by using the photochemical vapor deposition system can strongly improve the properties of the heteroepitaxially grown layer. The ability to reduce the electrical effects of the defects in InP on GaAs by hydrogenation may be able to serve as an intermediate step toward the growth of high quality devices. Here, it is worth emphasizing that the photochemical vapor deposition system should be of great interest because it does not damage the sample surface during hydrogenation.

\section{Acknowledgement}

This work is partly supported by the National Science Council of the Republic of China.

\section{References}

1 F. Riesz, K. Lischka, K. Rakennus, T. Hakkarainen and A. Pesek, J. Cryst. Growth, 114 (1991) 127.

2 G. Coudenys, I. Moerman and P. Demeester, J. Cryst. Growth, 114 (1991) 314.

3 T. Kimura, T. Kimura, E. Ishimura, F. Uesugi, M. Tsugami, K. Mizuguchi and T. Murotani, J. Cryst. Growth, 107 (1991) 827.

4 J. Leiber, A. Brauers, H. Heinecke, H. Luth and P. Balk, J. Cryst. Growth, 96 (1989) 483.

5 K. Bickmann and J. I lauck, Mater. Lett., 11 (1991) 236.

6 V. Swaminathan and A. T. Macrander (eds.), Materials Aspects of GaAs and InP Based Structures, Prentice Hall Advanced Reference Series, 1991, p. 213.

7 P. K. Willardson and A. C. Beer (eds.), Semiconductors and Semimetals, Vol. 21, Academic, New York, 1984.

8 S. J. Pearton, J. W. Corbett and T. S. Shi, Appl. Phys., A 43 (1987) 153.

9 E. E. Haller, Semicond. Sci. Technol., 6 (1991) 73.

10 S. J. Pearton, C. S. Wu, M. Stavola, F. Ren, J. Lopata, W. C. Dautremont-Smith, S. M. Vernon and V. E. Haven, Appl. Phys. Lett., 51 (1987) 496.

11 G. Bacguet, N. Lauret, M. Ben Marzouk, S. J. Pearton and W. S. Hobson, Solid State Commun., 80 (9) (1991) 669.

12 F. Ren, W. S. Hobson, S. J. Pearton, L. T. Dster and P. R. Smith, IEEE Electron Device Lett., 10 (1989) 389.

13 V. Swaminathan, U. K. Chakrabarti, W. S. Hobson, R. Caruso, J. Lopata, S. J. Pcarton and H. S. Luftman, J. Appl. Phys., $68(1990) 902$.

14 Y. F. Chen, C. S. Tsai, Y. H. Chang, Appl. Phys. Lett., 57 (1990) 70.

15 Y. F. Chen, W. S. Chen, S. H. Huang and F. Y. Juang, J. Appl. Phys., 69 (1991) 3360.

16 Y. F. Chen, K. C. Sung, W. K. Chen and Y. S. Lue, J. Appl. Phys., 71 (1) (1992) 509.

17 K. Yamasaki, M. Yoshida and T. Sugano, Jpn. J. Appl. Phys., 18 (1979) 113.

18 S. M. Sze, Physics of Semiconductor Devices, Wiley, New York, 2nd edn. 1981.

19 M. Neuberger, III-V Semiconducting compounds, Plenum, New York 1971.

20 O. Wada and A. Majerfeld, Electron Lett., 14 (1978) 125.

21 W. E. Spicer, T. Kendelewicz, N. Newman, K. K. Chin and I. Lindau, Surf. Sci., 168 (1986) 240. 